\title{
Asymptomatic Dengue Virus Infections, Cambodia, 2012-2013
}

\author{
Sowath Ly, ${ }^{1}$ Camille Fortas, ${ }^{1}$ Veasna Duong, Tarik Benmarhnia, Anavaj Sakuntabhai, Richard Paul, \\ Rekol Huy, Sopheak Sorn, Kunthy Nguon, Siam Chan, Souv Kimsan, Sivuth Ong, Kim Srorn Kim, \\ Sowathy Buoy, Lim Voeung, Philippe Dussart, Philippe Buchy, ${ }^{1,2}$ Arnaud Tarantola ${ }^{1}$
}

We investigated dengue virus (DENV) and asymptomatic DENV infections in rural villages of Kampong Cham Province, Cambodia, during 2012 and 2013. We conducted perifocal investigations in and around households for 149 DENV index cases identified through hospital and village surveillance. We tested participants $0.5-30$ years of age by using nonstructural 1 rapid tests and confirmed DENV infections using quantitative reverse transcription PCR or nonstructural 1-capture ELISA. We used multivariable Poisson regressions to explore links between participants' DENV infection status and household characteristics. Of 7,960 study participants, $346(4.4 \%)$ were infected with DENV, among whom $302(87.3 \%)$ were $<15$ years of age and $225(65.0 \%)$ were $\leq 9$ years of age. We identified $26(7.5 \%)$ participants with strictly asymptomatic DENV infection at diagnosis and during follow-up. We linked symptomatic DENV infection status to familial relationships with index cases. During the 2-year study, we saw fewer asymptomatic DENV infections than expected based on the literature.

A nnually, $\approx 390$ million people in $>100$ countries are infected with dengue virus (DENV); $70 \%$ of cases occur in countries in Asia (1). DENV is a flavivirus transmitted by Aedes aegypti and Ae. albopictus anthropophilic female mosquitoes. DENV has 4 distinct serotypes, DENV-1-4 (2); DENV infections can range from asymptomatic to life-threatening.

In Cambodia, the national dengue surveillance system reported 60,000 cases and 135 deaths attributed to DENV in 2012 and 2013 (3). Syndromic surveillance and random

Author affiliations: Institut Pasteur du Cambodge, Phnom Penh, Cambodia (S. Ly, C. Fortas, V. Duong, S. Sorn, K. Nguon,

S. Chan, S. Kimsan, S. Ong, P. Dussart, P. Buchy, A. Tarantola); University of California, San Diego, California, USA

(T. Benmarhnia); Institut Pasteur, Paris, France (A. Sakuntabhai,

R. Paul); Malaria National Center, Phnom Penh (R. Huy);

Kampong Cham Provincial Hospital, Kampong Cham, Cambodia

(K.S. Kim); Prey Chhor District Referral Hospital, Kampong Cham

(S. Buoy); Tboung Khmum District Referral Hospital, Thoung

Khmum, Cambodia (L. Voeung)

DOI: https://doi.org/10.3201/eid2507.181794 testing of dengue-like cases in referral pediatric hospitals in Cambodia likely underestimate the true disease burden (4). By definition, syndromic surveillance does not detect asymptomatic DENV infections, which increase vector transmission potential (5). Mammen et al. used both dengue-positive and dengue-negative index cases of febrile children to initiate perifocal investigations and found no cases in proximity to dengue-negative index cases (6). To maximize the number of recruited cases, we investigated homes around preidentified, dengue-positive index cases, as per a previous study (7). Our objectives were to document the proportion of strictly asymptomatic infections in this region of Cambodia; characterize human, sociodemographic, household-level, and mosquito control-related factors associated with DENV infection; and identify factors associated with asymptomatic DENV infection.

\section{Methods}

\section{Ethics Considerations}

The study protocol was approved by the Cambodian National Ethics Committee on Health Research. We obtained informed consent from participants or their guardians documented during hospital or village surveillance or perifocal investigations.

\section{Study Site}

We conducted a study in rural villages of Kampong Cham Province, $120 \mathrm{~km}$ northeast of Cambodia's capital, Phnom Penh. The study area included 368 villages with $\approx 60,000$ households and 3 hospitals within a $30-\mathrm{km}$ radius. Dengue is endemic in the region and mainly affects children $\leq 15$ years of age during the annual rainy season (June-October).

\section{Identification of Dengue Index Cases in Hospitals and Villages}

During June 1-October 31, 2012 and 2013, we identified DENV index cases in 3 referral hospitals and 26 villages

${ }^{1}$ These authors contributed equally to this article.

${ }^{2}$ Current affiliation: GlaxoSmithKline Vaccines Research and Design, Singapore. 
under active surveillance for febrile illness (5). We targeted persons $0.5-30$ years of age. In the 3 hospitals, blood samples were drawn at admission and discharge for all patients suspected of having DENV infection on the basis of clinical assessment and platelet count. In the 26 villages, volunteers monitored eligible residents weekly, measuring axillary body temperature using a digital thermometer to identify persons with temperatures $\geq 38^{\circ} \mathrm{C}$. Blood samples were drawn 1-2 days after fever onset, as described elsewhere $(4,8,9)$. All samples were screened for DENV infection by using a nonstructural (NS) $1 \mathrm{IgM} / \mathrm{IgG}$ combination rapid test. We confirmed DENV by using quantitative reverse transcription PCR (qRTPCR) or NS1-capture ELISA and included case-patients with confirmed DENV infection as index cases in the study.

\section{Perifocal Investigations}

Within 1-2 days of identifying an index case, whether from village or hospital surveillance, we began a perifocal investigation of the index case-patient's village of origin (7). For each perifocal investigation, we used a rapid dengue test kit to screen eligible residents in the index case-patient's household for DENV and completed a baseline questionnaire on individual symptoms, socioeconomic status, and household characteristics. We did the same in 20 households in a 100-meter radius of the index case-patient's household. We included persons $0.5-30$ years of age who consented or whose guarantor consented. We tested adults $>20$ years of age during the first year of the study but found no DENV-positive cases and did not test this age group during the second year. All consecutive cases were eligible for inclusion. To avoid bias through overlapping investigations of a potentially common source of infection, we did not conduct a perifocal investigation within 1 week of a previous investigation for $\geq 2$ index cases consecutively detected from the same village.

\section{DENV Testing and Case Definitions}

To screen for DENV infection during surveillance and perifocal investigations, investigators tested all blood samples on-site using SD BIOLINE Dengue Duo kit (Standard Diagnostics, https://www.alere.com), according to the manufacturer's instructions. Investigators interpreted results within 15-20 minutes and ruled out possible cases if the control band was negative. Blood samples from DENV-positive participants were sent to Institut Pasteur du Cambodge (Phnom Penh, Cambodia) for qRT-PCR testing, as described previously $(10,11)$, or confirmation using an NS1-capture ELISA $(11,12)$ with positive controls diluted to the limit of detection, negative, and nontemplate controls used during extraction and PCR steps to reduce inaccuracies (10). We considered cases confirmed when a blood sample tested positive by NS1 rapid test and was confirmed by NS1-capture ELISA or qRT-PCR. During the first year, we also tested participants for Japanese encephalitis virus (JEV) and chikungunya virus (CHIKV) IgM antibodies by ELISA and confirmed IgM-positive results using specific RT-PCR to ensure that symptoms were not related to CHIKV, JEV, or co-infections (11-13).

Symptomatic DENV-confirmed case-patients had fever, muscle or joint pain, rash, bleeding, prolonged headaches, or digestive signs. We asked participants whether they had taken antipyretics in the previous 24 hours. We termed afebrile all symptomatic DENV-positive participants without a fever and no antipyretic use. We considered participants asymptomatic when they had confirmed DENV infection, no antipyretic use, and no signs or symptoms, including fever. Participants who were symptomatic at initial diagnosis on day 0 received follow-up monitoring on days 2 and 7. We monitored asymptomatic participants daily on days $0-7$ and again on day 10 using a questionnaire to document signs and symptoms of DENV. In our analyses, we recategorized participants who were asymptomatic at baseline to symptomatic if they reported any symptoms during the follow-up period.

\section{Statistical Analysis}

We described DENV infection attack rates for perifocal investigations and the proportion of asymptomatic cases among all DENV infections and circulating serotypes. To explore participant- and household-level factors associated with DENV infection, we conducted a multivariable Poisson regression estimating attack rate ratios (ARRs) (14), excluding index cases. We built explanatory models around each participant-level and household-level factor, with and without adjusting for covariates. Participant-level factors included age, sex, occupation or schooling, and relationship to an index case-patient. Because we found collinearity between age and occupation, we adjusted only for age. We placed participants $0.5-1$ year of age into a specific category to account for differences in immunity and exposure to vectors due to reduced mobility. Household-level factors included the main source of income, source of water, measures against mosquitoes, and environmental factors favorable to mosquito development. We further divided the source of water into 2 categories: piped water (from indoor or outdoor taps with a tube well and pump) or nonpiped water (from a pond, river, lake, or a well without pump).

Considering the limited flight range of a female Aedes mosquito, we assumed that the probability of DENV transmission would be higher within a household than across households. To account for this factor and measure potential clustering, we developed a random-effects multilevel model. We computed the intraclass correlation coefficient as the proportion of the variability in the probability of infection attributable to differences between households versus differences within households (15). We excluded 
19 participants with missing covariates or predictors from the regression analyses. We explored associations between asymptomatic DENV infection and DENV serotype, participant-level factors, and the main source of income as socioeconomic indicators. We used the Fisher exact test for comparing proportions, the Student $t$ test for means, and an empty multilevel model to search for a cluster effect. We conducted analyses using Stata version 13 (StataCorp, https://www.stata.com).

\section{Results}

\section{Dengue Surveillance for Index Case Identification}

We identified 1,294 suspected DENV-infected persons, 834 (64.5\%) among hospital inpatients and 460 (35.5\%) through febrile illness surveillance in villages. Our testing confirmed 555 (66.5\%) DENV-positive cases among hospital patients and $36(7.8 \%)$ DENV-positive cases through febrile illness surveillance in villages.

\section{Perifocal Investigations}

From the 591 DENV-positive patients, we selected 149 (25.2\%) consecutive cases for which we conducted perifocal investigations: 131 from hospital patients, termed PI-H, and 18 from village febrile surveillance, termed PI-V. Perifocal investigations took place in 104 villages over the 2 rainy seasons and documented 7,960 participants, 6,811 (86\%) male and 1,149 (14\%) female, in 2,988 households (Figure).

We found $346(4.3 \%)$ persons who were positive for DENV infection, $225(65.0 \%)$ of whom were $<9$ years of age. We determined attack rates of 14.7/1,000 participants $(14 / 952)$ in PI-V and 47.4/1,000 $(332 / 7,008)$ in PI-H $(p<0.05)$. The attack rate over the 2 outbreak seasons increased marginally from $37 / 1,000$ persons $0.5-30$ years of age during the 2012 season to $46 / 1,000$ among those $0.5-$ 20 years of age during $2013(\mathrm{p}=0.056)$. Only $26(7.5 \%)$ of 346 DENV-positive participants remained strictly asymptomatic during the 10-day follow-up, an asymptomatic DENV-infection attack rate of 3.3/1,000 over the 2 years of our study. The proportion of asymptomatic infections was $21.4 \%$ (3/14) in PI-V and 6.9\% (23/332) in PI-H.

Besides headache and fever, symptomatic casepatients mainly experienced muscle, retro-orbital, and joint pain. Although fever is considered a typical symptom of DENV infection, careful interview, rigorous clinical assessment, and follow-up interviews showed that participants remained afebrile in 110 (31.8\%) of the 320 symptomatic DENV infections, even without antipyretics. Only $6(1.7 \%)$ of the DENV-positive case-patients required hospitalization, 2 with bleeding.

The 2 annual outbreaks were dominated by DENV-1. However, DENV-2 and DENV-4 emerged in 2013, and we detected DENV-3 sporadically (Table 1). During the first year of the study, samples from all symptomatic and asymptomatic DENV cases were negative for CHIKV by MAC-ELISA. Because we diagnosed no CHIKV in year 1 , and our national surveillance system also did not detect any CHIKV cases (data not shown), we did not perform CHIKV testing during year 2 . Of 26 asymptomatic cases confirmed by qRT-PCR or NS1-capture ELISA, 6 had positive JEV serology and also were positive for DENV IgM. We could not conclude whether JEV-positive results were indicative of a recent or acute JEV co-infection or the result of cross-reaction among flaviviruses. Among hospitalized patients, 2 had positive JEV results without detectable DENV IgM, even though qRT-PCR or NS1-capture ELISA was positive. These results could suggest a recent or acute JEV co-infection. During perifocal investigations, 42 participants tested positive for JEV by MAC-ELISA

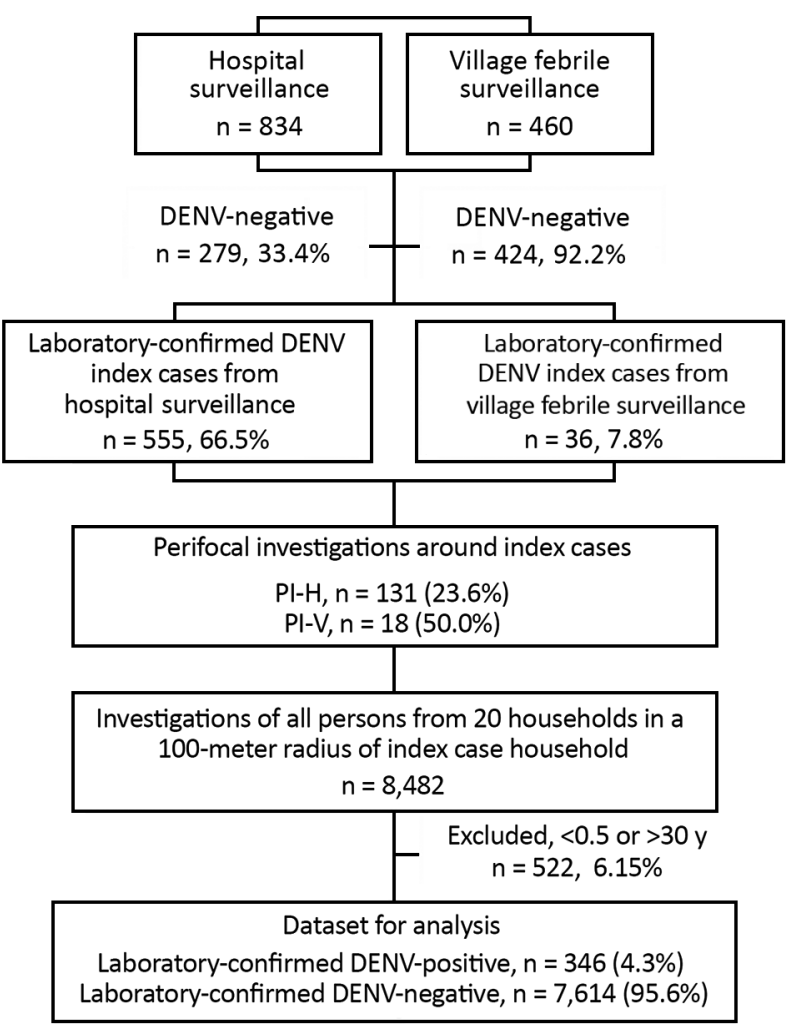

Figure. Participant screening and data flowchart for perifocal investigations for asymptomatic DENV infection, Cambodia, 2012-2013. Initial DENV screening of febrile cases was conducted using nonstructural (NS) $1 \mathrm{lgM} / \mathrm{lgG}$ combo rapid test. Perifocal investigations took place in villages of index cases; we screened all persons in 20 households within a 100-m radius of an index case household. We excluded persons $<0.5$ and $>30$ years of age. Laboratory confirmation of DENV was conducted through quantitative reverse transcription PCR or NS1-capture ELISA. DENV, dengue virus; $\mathrm{PI}-\mathrm{H}$, perifocal investigations conducted for index cases identified through hospital surveillance; PI-V, perifocal investigations conducted for index cases identified through village febrile surveillance. 
with negative DENV results, NS1, and qRT-PCR, supporting evidence of JEV co-circulation in the country (16).

Screened participants had a mean age $( \pm \mathrm{SD})$ of 11.7 $( \pm 7.9$; median 10; interquartile range 6-16); 6,207 (77.9\%) were schoolchildren, university students, or nonschooled children. The main sources of household income were planting crops $(61.0 \%)$, working in a factory $(14.3 \%)$, and keeping a shop (13.4\%). Participants reported low use of protective measures against mosquitoes, including mosquito coils in $787(26.3 \%)$ households, insecticide sprays in $557(18.6 \%)$ households, and larvicidal temephos in $374(12.5 \%)$ households. Our investigation found uncovered water jars in 1,867 (62.7\%) households and mosquito larvae in water containers of 1,663 $(55.7 \%)$ households (Table 2).

Among DENV-positive cases, boys and girls were equally affected at a mean $( \pm \mathrm{SD})$ age of $8.5( \pm 5.7)$ years. Compared with persons 15-30 years of age, we found that children 1-10 years of age had a higher ARR of DENV infection (ARR 4.04 [95\% CI 2.72-5.98] for those 1-5 years of age and ARR 3.83 [95\% CI 2.59-5.67] for those 6-10 years of age). Siblings and cousins of index casepatients were more prone to DENV infection than neighbors were; siblings were 2.24 (95\% CI 1.42-3.53) times and cousins 1.40 (95\% CI 1.02-1.90) times more at risk for infection than neighbors. Participants who used piped water had a higher risk for DENV infection (ARR 1.35 [95\%
CI 1.06-1.71]) than did those who used nonpiped water. Households in which the main source of income was fishing, farming, or animal husbandry also had higher risks for infection (ARR 2.02 [95\% CI 1.18-3.45]). Households reporting mosquito control-related parameters did not have a lower risk for DENV infection (Table 2).

The main source of income was similarly distributed between households with $\geq 1$ case and households with no cases $(p=0.272)$. Our multilevel model showed a notable clustering effect at the household level after adjustment (intraclass correlation coefficient $40.8 \%$ ).

We found $26(7.5 \%)$ case-patients, $17(65.4 \%)$ male and $9(34.6 \%)$ female, who were positive for DENV infection but remained asymptomatic. We found serotypes DENV-1, DENV-2, and DENV-4 in our study group (Table 3). We used a multilevel approach to explore the role of specific serotypes and participant-level factors, such as age, gender, and relationship to the index case-patient, a proxy for common genetic background, with being DENVpositive and asymptomatic. We found that only family relationship to the index case-patient was associated with asymptomatic infection. We did not identify a cluster effect or associated factors.

\section{Discussion}

We screened 7,960 participants in communities in Cambodia during 2012 and 2013 and found 346 (4.3\%) participants

\begin{tabular}{|c|c|c|c|}
\hline Surveillance data & 2012 & 2013 & Total \\
\hline No. participants & 2,391 & 5,569 & 7,960 \\
\hline No. villages investigated & 35 & 77 & 104 \\
\hline No. perifocal investigations conducted & 47 & 102 & 149 \\
\hline Mean no. participants per perifocal investigation & 51 & 55 & 53 \\
\hline Confirmed infections, no. (\%) & 88 & 258 & 346 \\
\hline Strictly asymptomatic & $5(5.7)$ & $21(8.1)$ & $26(7.5)$ \\
\hline Afebrile & $33(37.5)$ & $77(29.8)$ & $110(31.2)$ \\
\hline Symptomatic & $83(94.3)$ & $237(91.9)$ & $320(92.5)$ \\
\hline Attack rate/1,000 participants, $\%$ & 36.8 & 46.3 & 43.5 \\
\hline Asymptomatic infections & 2.1 & 3.8 & 3.3 \\
\hline Symptomatic infections & 34.7 & 42.6 & 40.2 \\
\hline Afebrile infections & 13.8 & 13.8 & 13.8 \\
\hline Symptoms at diagnosis or follow-up, no. (\%) & 83 & 237 & 320 \\
\hline Fever & $55(66.2)$ & $180(75.9)$ & $236(73.8)$ \\
\hline Headache & $52(62.7)$ & 169 (71.3) & $221(69.1)$ \\
\hline Muscle pain & $16(19.3)$ & $73(30.8)$ & $89(27.8)$ \\
\hline Retro-orbital pain & $17(20.5)$ & $73(30.8)$ & $90(28.1)$ \\
\hline Joint pain & $17(20.5)$ & $68(28.7)$ & $85(26.5)$ \\
\hline Rash & $15(18.1)$ & $53(22.4)$ & $68(21.3)$ \\
\hline Any bleeding & $13(15.7)$ & $50(21.1)$ & $63(19.7)$ \\
\hline Hospitalizations, no. (\%) & $3(3.5)$ & $8(3.3)$ & $11(3.3)$ \\
\hline DENV infections & 88 & 258 & 346 \\
\hline \multicolumn{4}{|l|}{ Serotype, no. (\%) } \\
\hline DENV-1 & $82(98.8)$ & $188(72.9)$ & $270(78.0)$ \\
\hline DENV-2 & $1(1.2)$ & $36(13.9)$ & $37(10.7)$ \\
\hline DENV-3 & 0 & $2(0.8)$ & $2(0.6)$ \\
\hline DENV-4 & 0 & $31(12.0)$ & $31(9.0)$ \\
\hline DENV-1 and DENV-2 & 0 & $1(0.4)$ & $1(0.3)$ \\
\hline Missing & 5 & 0 & 5 \\
\hline
\end{tabular}

*Participants 0.5-30 years of age in 2012 and 0.5-20 years of age in 2013. DENV, dengue virus. 
infected by DENV; 26 (7.5\%) remained asymptomatic before, during, and after our study. We found comparable attack rates, 37/1,000 persons in 2012 and 46/1,000 persons in 2013, to other community investigations conducted in Cambodia. For instance, another study reported DENV attack rates of 13.4-57.8 cases/1,000 persons during 2006-2008 (4). Previous studies only included participants $\leq 20$ years of age, but we included persons $0.5-30$ years of age with confirmed DENV infection, even symptomatic but afebrile case-patients, who were $31.8 \%$ of the DENV infections in

Table 2. Participant and household characteristics with unadjusted and adjusted attack rate ratios for factors potentially associated with dengue virus infection, Cambodia, 2012-2013*

\begin{tabular}{|c|c|c|c|c|c|}
\hline Characteristics & Infected & Uninfected & Total & $\begin{array}{c}\text { Unadjusted ARR } \\
(95 \% \mathrm{Cl})\end{array}$ & $\begin{array}{l}\text { Adjusted ARR } \\
(95 \% \mathrm{Cl})\end{array}$ \\
\hline Participants & 346 & 7,614 & 7,960 & & \\
\hline \multicolumn{6}{|l|}{ Sex } \\
\hline $\mathrm{M}$ & 171 & 4,103 & 4,272 & Referent & Referent \\
\hline $\mathrm{F}$ & 175 & 3,511 & 3,686 & $1.14(0.92-1.40)$ & $1.01(0.82-1.24)$ \\
\hline \multicolumn{6}{|l|}{ Age, y† } \\
\hline $0.5-<1$ & $9(2.6)$ & $150(2.0)$ & $159(2.0)$ & $3.47(1.65-7.32)$ & $3.53(1.67-7.46)$ \\
\hline $1-<5$ & $108(31.2)$ & $1,701(22.3)$ & $1,809(22.7)$ & $3.98(2.69-5.90)$ & $4.04(2.72-5.98)$ \\
\hline $5-<10$ & $126(36.4)$ & $2,083(27.4)$ & $2,209(27.8)$ & $3.79(2.56-5.60)$ & $3.83(2.59-5.67)$ \\
\hline $10-<15$ & $71(20.5)$ & $1,675(22.0)$ & $1,746(21.9)$ & $2.59(1.70-3.94)$ & $2.55(1.67-3.88)$ \\
\hline $15-30$ & $32(9.3)$ & 2,005 (26.3) & 2,037 (25.6) & Referent & Referent \\
\hline Mean ( \pm SD, median) & $8.5( \pm 5.7,7)$ & $11.9( \pm 8.0,10)$ & $11.7( \pm 7.9,10)$ & - & - \\
\hline \multicolumn{6}{|l|}{ Occupation $\ddagger$} \\
\hline Student, school or university & $171(49.8)$ & $3,588(47.2)$ & $3,759(47.3)$ & $2.14(1.35-3.41)$ & $2.14(1.34-3.41)$ \\
\hline Preschool or unschooled & $149(43.2)$ & $2,299(30.2)$ & $2,448(30.8)$ & $2.84(1.79-4.54)$ & $2.84(1.78-4.54)$ \\
\hline Planting crops & $20(5.8)$ & $910(12.0)^{\prime}$ & $930(11.7)$ & Referent & Referent \\
\hline Other & $5(1.5)$ & $809(10.6)$ & $814(10.2)$ & $0.28(0.10-7.76)$ & $0.28(0.10-7.76)$ \\
\hline Missing & $1(0.2)$ & $8(0.1)$ & $9(1.1)$ & & \\
\hline \multicolumn{6}{|l|}{ Relationship to index case-patient§ } \\
\hline Neighbor & $260(75.4)$ & $6,309(83.0)$ & $6,569(82.6)$ & Referent & Referent \\
\hline Cousin & $58(16.8)$ & $991(13.0)$ & $1,049(13.2)$ & $1.38(1.01-1.89)$ & $1.40(1.02-1.90)$ \\
\hline Sibling & $23(6.7)$ & $251(3.3)$ & $274(3.5)$ & $2.11(1.33-3.34)$ & $2.24(1.42-3.53)$ \\
\hline Other & $5(1.2)$ & $55(0.7)$ & $59(0.7)$ & $1.66(0.59-4.65)$ & $1.76(0.34-4.90)$ \\
\hline Missing & $1(0.2)$ & $8(0.1)$ & $9(1.1)$ & & \\
\hline Households & $292 \rrbracket$ & 2,706 & 2,988 & & \\
\hline \multicolumn{6}{|l|}{ Water source\# } \\
\hline Nonpiped & $108(36.3)$ & $1,186(43.7)$ & $1,284(43.0)$ & Referent & Referent \\
\hline Piped & $184(63.7)$ & $1,520(56.3)$ & $1,704(57.0)$ & $1.32(1.03-1.69)$ & $1.35(1.06-1.71)$ \\
\hline \multicolumn{6}{|l|}{ Primary source of income ${ }^{* *}$} \\
\hline Planting crops & $176(60.9)$ & $1,648(61.0)$ & $1,824(61.0)$ & Referent & Referent \\
\hline Working in a factory & $42(14.5)$ & $384(14.2)$ & $426(14.3)$ & $1.16(0.84-1.62)$ & $1.20(0.87-1.66)$ \\
\hline Shopkeeping & $37(12.8)$ & $362(13.4)$ & 399 (13.4) & $0.97(0.67-1.40)$ & $1.03(0.72-1.48)$ \\
\hline Fishing, farming, animal husbandry & $14(4.8)$ & $55(2.0)$ & $69(2.3)$ & $1.98(1.15-3.43)$ & $2.02(1.18-3.45)$ \\
\hline Working in government & $5(1.7)$ & $57(2.1)$ & $62(2.1)$ & $0.94(0.38-2.30)$ & $0.99(0.41-2.37)$ \\
\hline Other & $15(5.2)$ & $193(7.2)$ & $208(7.0)$ & $0.76(0.43-1.32)$ & $0.85(0.50-1.46)$ \\
\hline \multicolumn{6}{|l|}{ Mosquito control measures†† } \\
\hline Temephos & $26(9.0)$ & $348(12.9)$ & $374(12.5)$ & $0.70(0.47-1.06)$ & $0.73(0.48-1.10)$ \\
\hline Larvivorous fish & $26(9.0)$ & $214(7.9)$ & $240(8.3)$ & $1.14(0.75-1.74)$ & $1.18(0.78-1.79)$ \\
\hline Treated mosquito netting & $27(9.3)$ & $311(11.5)$ & $338(11.3)$ & $0.78(0.52-1.17)$ & $0.82(0.55-1.21)$ \\
\hline Treated jar cover & $3(1.0)$ & $47(1.7)$ & $50(1.7)$ & $0.73(0.24-2.24)$ & $0.77(0.26-2.27)$ \\
\hline Coils & 77 (26.6) & $710(26.3)$ & $787(26.3)$ & $1.08(0.82-1.41)$ & $1.16(0.89-1.51)$ \\
\hline Insecticide spray & 44 (15.2) & $513(19.0)$ & $557(18.6)$ & $0.79(0.57-1.10)$ & $0.88(0.63-1.22)$ \\
\hline \multicolumn{6}{|l|}{ Environmental factors ${ }^{* *}$} \\
\hline Vegetable garden & $57(9.7)$ & $546(20.2)$ & $603(20.2)$ & $0.89(0.66-1.21)$ & $0.89(0.66-1.20)$ \\
\hline Water collection around house & $126(43.6)$ & $1,180(43.7)$ & $1,306(44.7)$ & $0.91(0.71-1.15)$ & $0.88(0.70-1.12)$ \\
\hline Uncovered water jars & $178(61.6)$ & $1,689(62.6)$ & $1,867(62.5)$ & $0.96(0.75-1.22)$ & $0.97(0.76-1.23)$ \\
\hline Larvae in water containers & $168(58.1)$ & $1,495(55.4)$ & $1,663(55.7)$ & $1.09(0.86-1.39)$ & $1.07(0.85-1.37)$ \\
\hline $\begin{array}{l}\text { Distance from house to nearest } \\
\text { water jar, } m( \pm S D)\end{array}$ & $1.5( \pm 2.2)$ & $1.3( \pm 2.0)$ & $1.3( \pm 2.0)$ & $1.00(0.98-1.02)$ & $1.00(0.98-1.02)$ \\
\hline Missing for all items & 3 & 7 & 10 & & \\
\hline
\end{tabular}

*Values are no. or no. (\%) except as indicated. ARR, attack rate ratio; DENV, dengue virus.

†Participants $0.5-30$ years of age in 2012 and $0.5-20$ years of age in 2013

$\ddagger$ Adjusted for sex.

§Adjusted for age.

INo. housesholds with $>1$ DENV case.

\#Nonpiped water comes from a river, pond, lake, or a well that does not have a pump; piped water comes from indoor or outdoor taps with a tube well and pump.

**Adjusted for sex and occupation.

††Adjusted for age, relationship to index case-patient, occupation, and primary source of income. 
Table 3. Univariate tests for associations between sociodemographic factors and infecting serotypes with asymptomatic dengue virus infections, Cambodia, 2012-2013*

\begin{tabular}{|c|c|c|c|}
\hline Factor & $\begin{array}{c}\text { Asymptomatic, } \\
\mathrm{n}=26\end{array}$ & $\begin{array}{c}\text { Symptomatic, } \\
n=320\end{array}$ & $\mathrm{p}$ value \\
\hline \multicolumn{4}{|l|}{ Sex } \\
\hline M & 17 & 154 & \multirow[t]{2}{*}{0.09} \\
\hline $\mathrm{F}$ & 9 & 166 & \\
\hline \multicolumn{4}{|l|}{ Age, y } \\
\hline 0.5 to $<1$ & 0 & $9(2.8)$ & \multirow[t]{7}{*}{0.976} \\
\hline $1-5$ & $9(34.6)$ & $99(30.9)$ & \\
\hline $6-10$ & $9(34.6)$ & $117(36.6)$ & \\
\hline $11-14$ & $5(19.2)$ & $66(20.6)$ & \\
\hline $15-30$ & $3(11.5)$ & $29(9.1)$ & \\
\hline Mean & & 11.0 & \\
\hline (+ SD, median) & $(+7.2,8)$ & $(+7.1,10)$ & \\
\hline \multicolumn{4}{|c|}{ Relationship to index case-patient } \\
\hline Neighbor & $17(65.4)$ & $243(76.0)$ & \multirow[t]{4}{*}{0.004} \\
\hline Cousin & $5(19.2)$ & $53(16.6)$ & \\
\hline Sibling & $1(3.9)$ & $22(6.9)$ & \\
\hline Other & $3(11.5)$ & $1(0.3)$ & \\
\hline \multicolumn{4}{|c|}{ Source of household income } \\
\hline Planting crops & $14(53.8)$ & $192(60.0)$ & \multirow[t]{6}{*}{0.812} \\
\hline $\begin{array}{l}\text { Working in a } \\
\text { factory }\end{array}$ & $3(11.5)$ & $53(16.6)$ & \\
\hline Shopkeeping & $5(19.2)$ & $36(11.3)$ & \\
\hline $\begin{array}{l}\text { Fishing, farming, } \\
\text { animal husbandry }\end{array}$ & $1(3.9)$ & $17(5.3)$ & \\
\hline $\begin{array}{l}\text { Working in } \\
\text { government }\end{array}$ & 0 & $6(1.9)$ & \\
\hline Other or missing & $3(11.5)$ & $16(5.0)$ & \\
\hline \multicolumn{4}{|l|}{ DENV serotype $\ddagger$} \\
\hline DENV-1 & $21(80.8)$ & $249(79.1)$ & \multirow[t]{4}{*}{0.892} \\
\hline DENV-2 & $2(7.7)$ & $35(11.1)$ & \\
\hline DENV-3 & 0 & $2(0.6)$ & \\
\hline DENV-4 & $3(11.5)$ & $28(8.9)$ & \\
\hline
\end{tabular}

"Values are no. (\%) patients except as indicated. DENV, dengue virus. †By Fisher test or $\chi^{2}$ test.

‡Data for 5 symptomatic patients were missing, and another patient was excluded from analysis because of co-infection with DENV-1 and DENV-2.

this study. We noted that attack rates were lower in PI-V, 14.7/1,000 participants (14/952), than in PI-H, 47.4/1,000 participants $(332 / 7,008)$. Circulation of DENV around febrile index case-patients identified through PI-V was less intense, but with more asymptomatic cases, than around index case-patients identified through PI-H. Aside from possible detection biases (17), multiple factors could explain this observation and deserve further research.

Our study documented cases of DENV infection in transmission clusters located around index case-patients. We found that $26.6 \%$ of DENV-confirmed case-patients reported clinical symptoms, including headache and muscle pain, but no fever even in the absence of antipyretics, comparable to data from Thailand, where $40.4 \%$ of the DENV cases remained afebrile (17). The appearance of afebrile DENV-infected patients raises potential concerns for case definitions for detection, especially of imported cases in at-risk countries.

An additional $7.5 \%$ of DENV-confirmed case-patients had no symptoms during the 10-day course of clinical monitoring, a considerably lower rate than estimates from other prospective studies $(5,18-21)$. Published sources refer to inapparent infections, often defined as afebrile clinical complaints with biologic evidence of DENV infection, ranging from $20 \%$ to $80 \%$ of cases $(19,22,23)$. Previous studies used different definitions of asymptomatic infection than ours, but the major difference lies in follow-up monitoring. Other retrospective studies used school or work absenteeism as a basis for follow-up (19). Strictly asymptomatic patients, such as those we describe, escape detection by surveillance or control measures, infect mosquitoes, and might disproportionately contribute to DENV transmission (5).

The DENV burden documented through hospitalbased surveillance of febrile case-patients in Thailand and Vietnam showed a shift to older age groups $(24,25)$. Our active, systematic case-finding system to identify DENV in villages in Cambodia found the attack rate was highest in children $<10$ years of age, which is what we expected in a dengue-endemic country with frequent outbreaks demonstrated in other careful studies (26). This finding raises concerns because recommendations for the only licensed dengue vaccine are for use in persons 9-45 years of age with demonstrated evidence of past DENV infection (27). Our study demonstrates that children in rural Cambodia might have undergone $>1$ DENV infection before 9 years of age, reducing the potential cost-effectiveness of vaccination.

Few studies have explored the role of socioeconomic status, which might be a proxy for peridomestic environmental management, on DENV infection in Southeast Asia. Often, the direction of the association is unclear and socioeconomic status has entirely different associations depending on the setting (28). Our study shows that the adjusted risk for DENV infection was highest in households in which the main source of income was from fishing, farming, or animal husbandry, activities associated with lower average household income in Cambodia.

We found temephos provided no additional protection against DENV infection after adjusting for other factors. Although temephos is effective in reducing Aedes spp. larval populations in water storage jars, its use did not correlate with lower DENV transmission in Cambodia or elsewhere (8), due to incorrect distribution coverage, dosage, and placement (29) or multiple vector breeding sites. In addition, increases in temephos-resistant $A$. aegypti mosquito larvae have been documented in Cambodia (30).

Unexpectedly, we found a higher risk for DENV with piped water as a main water source after adjusting for other factors, contrary to a study in Thailand (6). However, piped water with suboptimal sanitation in Cambodia might contribute to collection of water in or around households that could become breeding sites for DENV-transmitting mosquitoes.

We found that $40.8 \%$ of the variability in probability of being DENV infected was explained by differences between households. Those living in the same household as an index case-patient were 2.11 times more likely to be 
infected, consistent with other published sources. A study in Mexico found that the risk for infection for those living with an index case-patient was twice that of someone living in a 50-meter radius of an index case-patient (31). This relationship was further described in a cluster study in Thailand that showed decreased risk for infection with increasing distance from the index case household (31). This clustering effect around an index case, however, seems to occur only on a short temporal scale, at least in urban settings (32).

Rates and severity of illness after infection with the different DENV serotypes differ widely $(33,34)$. The only notable epidemiologic factor associated with asymptomatic DENV infections in our study was being family-related to the index case-patient. Another study showed that adaptive immune responses against DENV differ between persons with symptomatic and asymptomatic DENV infection (35), which might explain our observations. We found no other associated epidemiologic factor, including age or cluster effects. Although the ratio of male to female participants was twice as high among asymptomatic than symptomatic participants, this finding was not statistically significant, likely due to sample size.

Although the size and duration of our study confer strength to our data, it might suffer from biases and limitations, especially due to the small number of strictly asymptomatic DENV-positive participants after stratifying by DENV serotype. We found dengue incidence rates highest in young children. These data might be biased because we focused on investigating clusters around an index case, perhaps overestimating the incidence in the general population. DENV circulation, however, is intense in children in Cambodia, and these figures remain comparable to those found in dengue studies that use different methods, ranging from 20 to 80 per 1,000 person-seasons (1). Furthermore, we did not capture cases referred to the private sector, lowering our estimates somewhat. Healthy male workers often were away at the time of the investigations, possibly leading to an overestimation of DENV incidence. These workers, however, are $\geq 18$ years of age, but DENV infections occur mainly in persons $<15$ years of age in Cambodia (4).

Documentation bias might also have pulled our risk factor estimates toward the null. We did not document solid waste disposal in our study, but comparatively high Breteau index values have been reported in Cambodia (29). In addition, we could have missed details or misrepresented implementation of mosquito-control measures. Despite the potential misclassification, mosquito-control measures remain nondifferential and likely had no major effect on our risk estimates.

Further, $7.5 \%$ of our DENV-infected participants remained strictly asymptomatic. Aside from case definition issues we discuss, our method of screening for DENV around symptomatic cases might have underestimated the number of asymptomatic DENV infections. In addition, we did not enroll persons who tested negative for DENV IgM, NS1-capture ELISA, and qRT-PCR. Some of these persons might have been infected but not yet mounted an IgM response, so that NS1 and viral RNA titers had already receded to undetectable levels when we tested them. This strict case definition might have underestimated the incidence of asymptomatic cases, but a precise retrospective documentation of such cases would be extremely difficult. Similarly, we retrospectively conducted MACELISA on samples collected during perifocal investigations and identified 11 cases of IgM seroconversion in the absence of PCR- or NS1-positive tests. Even in the context of JEV cocirculation, some of these cases could have been true DENV infections, but including them would not have changed the overall estimated attack rate. Previous studies suggested virus serotype might affect severity and types of symptoms and observed that DENV-1 infections more frequently were associated with clinically apparent illness $(36,37)$. Virus molecular analysis studies are ongoing to determine whether specific strains cause more asymptomatic infection than others. Furthermore, DENV infection in Cambodia occurs mainly in children who might be more likely to answer positively to daily-repeated questions on dengue symptoms, somewhat underestimating asymptomatic cases. Having implemented careful and thorough 10day clinical assessment of objective symptoms in each asymptomatic DENV-positive participant, we believe these figures reflect the true proportion of strictly asymptomatic DENV infection in our setting. However, we collected our findings mainly in children with DENV-1 infection in Cambodia. Whether these findings are directly applicable to other epidemiologic settings, populations, or virus serotypes or genotypes remains to be determined (33).

Finally, vaccination against JEV might have led to cross-protection against symptomatic dengue. Data on JEV vaccination were not collected during perifocal investigations. According to local health centers, however, JEV vaccine has been provided only recently and only for children 9-24 months of age. In our study, only 3 children were DENV-positive in that age category.

Our study demonstrates that systematically relying on fever for DENV case definition can underestimate cases and hinder control efforts in areas with potential vectors and at risk for DENV introduction. We found $7.5 \%$ of DENV-infected participants remained strictly asymptomatic, which has wide-ranging epidemiologic consequences. Undetected sources can increase transmission (5), a factor that must be taken into account in future vaccine coverage and vaccine effectiveness studies. The attack rate differences observed around febrile index case-patients detected in village surveillance and index case-patients detected in 
hospital surveillance deserve further study. In-depth virus (36) and human genetic studies could contribute useful insights $(33,35)$. Our strict definition of asymptomatic DENV infections should be considered when designing studies that aim to elucidate the pathophysiological mechanisms of dengue disease.

\section{Acknowledgments}

The authors gratefully acknowledge participating hospitals, villages, and study participants, as well as Tineke Cantaert for her editorial comments and suggestions.

Dengue Framework for Resisting Epidemics in Europe studies were funded by a grant (no. 282378) from the European Union 7th FP.

P.B. is a former head of virology at Institut Pasteur du Cambodge and is an employee of GSK Vaccines, Singapore.

\section{About the Authors}

Dr. Ly is a medical doctor and epidemiologist at the Epidemiology \& Public Health Department at Institut Pasteur du Cambodge; his primary research interests are epidemiology of endemic and epidemic arboviruses and zoonoses in the Mekong Region. Ms. Fortas is an epidemiologist whose research interests are tropical infectious diseases in low- and middleincome countries.

\section{References}

1. Bhatt S, Gething PW, Brady OJ, Messina JP, Farlow AW, Moyes CL, et al. The global distribution and burden of dengue. Nature. 2013;496:504-7. http://dx.doi.org/10.1038/nature12060

2. Simmons CP, Farrar JJ, van Vinh Chau N, Wills B. Dengue. N Engl J Med. 2012;366:1423-32. http://dx.doi.org/10.1056/ NEJMra1110265

3. Western Pacific Regional Office of the World Health Organization. Dengue situation updates [cited $2017 \mathrm{Jul}$ 12]. http://www.wpro. who.int/entity/emergencies/wp_dengue biweekly/en

4. Vong S, Goyet S, Ly S, Ngan C, Huy R, Duong V, et al. Under-recognition and reporting of dengue in Cambodia: a capturerecapture analysis of the National Dengue Surveillance System. Epidemiol Infect. 2012;140:491-9. http://dx.doi.org/10.1017/ S0950268811001191

5. Duong V, Lambrechts L, Paul RE, Ly S, Lay RS, Long KC, et al. Asymptomatic humans transmit dengue virus to mosquitoes. Proc Natl Acad Sci U S A. 2015;112:14688-93. http://dx.doi.org/ 10.1073/pnas. 1508114112

6. Mammen MP, Pimgate C, Koenraadt CJM, Rothman AL, Aldstadt J, Nisalak A, et al. Spatial and temporal clustering of dengue virus transmission in Thai villages. PLoS Med. 2008;5:e205. http://dx.doi.org/10.1371/journal.pmed.0050205

7. Dussart P, Baril L, Petit L, Beniguel L, Quang LC, Ly S, et al. Clinical and virological study of dengue cases and the members of their households: the multinational DENFRAME Project. PLoS Negl Trop Dis. 2012;6:e1482. http://dx.doi.org/10.1371/ journal.pntd.0001482

8. Huy R, Buchy P, Conan A, Ngan C, Ong S, Ali R, et al. National dengue surveillance in Cambodia 1980-2008: epidemiological and virological trends and the impact of vector control. Bull
World Health Organ. 2010;88:650-7. http://dx.doi.org/10.2471/ BLT.09.073908

9. Vong S, Khieu V, Glass O, Ly S, Duong V, Huy R, et al. Dengue incidence in urban and rural Cambodia: results from populationbased active fever surveillance, 2006-2008. PLoS Negl Trop Dis. 2010;4:e903. http://dx.doi.org/10.1371/journal.pntd.0000903

10. Hue KDT, Tuan TV, Thi HTN, Bich CTN, Anh HHL, Wills BA, et al. Validation of an internally controlled one-step real-time multiplex RT-PCR assay for the detection and quantitation of dengue virus RNA in plasma. J Virol Methods. 2011;177:168-73. http://dx.doi.org/10.1016/j.jviromet.2011.08.002

11. Andries A-C, Duong V, Ngan C, Ong S, Huy R, Sroin KK, et al. Field evaluation and impact on clinical management of a rapid diagnostic kit that detects dengue NS1, IgM and IgG. PLoS Negl Trop Dis. 2012;6:e1993. http://dx.doi.org/10.1371/ journal.pntd.0001993

12. Andries A-C, Duong V, Ong S, Ros S, Sakuntabhai A, Horwood P, et al. Evaluation of the performances of six commercial kits designed for dengue NS1 and anti-dengue IgM, IgG and IgA detection in urine and saliva clinical specimens. BMC Infect Dis. 2016;16:201. http://dx.doi.org/10.1186/s12879-016-1551-x

13. Duong V, Andries A-C, Ngan C, Sok T, Richner B, Asgari-Jirhandeh N, et al. Reemergence of chikungunya virus in Cambodia. Emerg Infect Dis. 2012;18:2066-9. http://dx.doi.org/ 10.3201/eid1812.120471

14. Zou G. A modified Poisson regression approach to prospective studies with binary data. Am J Epidemiol. 2004;159:702-6. http://dx.doi.org/10.1093/aje/kwh090

15. Wu S, Crespi CM, Wong WK. Comparison of methods for estimating the intraclass correlation coefficient for binary responses in cancer prevention cluster randomized trials. Contemp Clin Trials. 2012;33:869-80. http://dx.doi.org/10.1016/j.cct.2012.05.004

16. Tarantola A, Goutard F, Newton P, de Lamballerie X, Lortholary O, Cappelle J, et al. Estimating the burden of Japanese encephalitis virus and other encephalitides in countries of the Mekong region. PLoS Negl Trop Dis. 2014;8:e002533. https://doi.org/10.1371/ journal.pntd.0002533

17. Yoon I-K, Srikiatkhachorn A, Hermann L, Buddhari D, Scott TW, Jarman RG, et al. Characteristics of mild dengue virus infection in Thai children. Am J Trop Med Hyg. 2013;89:1081-7. http://dx.doi.org/10.4269/ajtmh.13-0424

18. Porter KR, Beckett CG, Kosasih H, Tan RI, Alisjahbana B, Rudiman PIF, et al. Epidemiology of dengue and dengue hemorrhagic fever in a cohort of adults living in Bandung, West Java, Indonesia. Am J Trop Med Hyg. 2005;72:60-6. http://dx.doi.org/10.4269/ajtmh.2005.72.60

19. Endy TP, Chunsuttiwat S, Nisalak A, Libraty DH, Green S, Rothman AL, et al. Epidemiology of inapparent and symptomatic acute dengue virus infection: a prospective study of primary school children in Kamphaeng Phet, Thailand. Am J Epidemiol. 2002;156:40-51. http://dx.doi.org/10.1093/aje/kwf005

20. Kosasih H, Alisjahbana B, Nurhayati, de Mast Q, Rudiman IF, Widjaja S, et al. The epidemiology, virology and clinical findings of dengue virus infections in a cohort of Indonesian adults in western Java. PLoS Negl Trop Dis. 2016;10:e0004390. http://dx.doi.org/10.1371/journal.pntd.0004390

21. Yoon I-K, Rothman AL, Tannitisupawong D, Srikiatkhachorn A, Jarman RG, Aldstadt J, et al. Underrecognized mildly symptomatic viremic dengue virus infections in rural Thai schools and villages. J Infect Dis. 2012;206:389-98. http://dx.doi.org/10.1093/infdis/jis357

22. Balmaseda A, Standish K, Mercado JC, Matute JC, Tellez Y, Saborío $\mathrm{S}$, et al. Trends in patterns of dengue transmission over 4 years in a pediatric cohort study in Nicaragua. J Infect Dis. 2010;201:5-14. http://dx.doi.org/10.1086/648592

23. Endy TP, Anderson KB, Nisalak A, Yoon I-K, Green S, Rothman AL, et al. Determinants of inapparent and symptomatic 
dengue infection in a prospective study of primary school children in Kamphaeng Phet, Thailand. PLoS Negl Trop Dis. 2011;5:e975. http://dx.doi.org/10.1371/journal.pntd.0000975

24. Anders KL, Nga H, Thuy NTV, Ngoc TV, Tam CT, Tai LTH, et al. Households as foci for dengue transmission in highly urban Vietnam. PLoS Negl Trop Dis. 2015;9:e0003528. http://dx.doi.org/10.1371/journal.pntd.0003528

25. Limkittikul K, Brett J, L'Azou M. Epidemiological trends of dengue disease in Thailand (2000-2011): a systematic literature review. PLoS Negl Trop Dis. 2014;8:e3241. http://dx.doi.org/ 10.1371/journal.pntd.0003241

26. Hadinegoro SR, Arredondo-García JL, Capeding MR, Deseda C, Chotpitayasunondh T, Dietze R, et al.; CYD-TDV Dengue Vaccine Working Group. Efficacy and long-term safety of a dengue vaccine in regions of endemic disease. N Engl J Med. 2015;373:1195-206. http://dx.doi.org/10.1056/NEJMoa1506223

27. Dengue vaccine: WHO position paper, September 2018 recommendations. Vaccine. 2018;S0264-410X(18)31339-2

28. Mulligan K, Dixon J, Joanna Sinn C-L, Elliott SJ. Is dengue a disease of poverty? A systematic review. Pathog Glob Health. 2015; 109:10-8. http://dx.doi.org/10.1179/2047773214Y.0000000168

29. Khun S, Manderson LH. Abate distribution and dengue control in rural Cambodia. Acta Trop. 2007;101:139-46. http://dx.doi.org/ 10.1016/j.actatropica.2007.01.002

30. Polson KA, Curtis C, Seng CM, Olson JG, Chantha N, Rawlins SC. Susceptibility of two Cambodian populations of Aedes aegypti mosquito larvae to temephos during 2001. Dengue Bull. 2001; 25:79-83.

31. Martínez-Vega RA, Danis-Lozano R, Díaz-Quijano FA, Velasco-Hernández J, Santos-Luna R, Román-Pérez S, et al. Peridomestic infection as a determining factor of dengue transmission. PLoS Negl Trop Dis. 2015;9:e0004296. http://dx.doi.org/10.1371/journal.pntd.0004296

32. Yoon I-K, Getis A, Aldstadt J, Rothman AL, Tannitisupawong D, Koenraadt CJM, et al. Fine scale spatiotemporal clustering of dengue virus transmission in children and Aedes aegypti in rural Thai villages. PLoS Negl Trop Dis. 2012;6:e1730. http://dx.doi.org/ 10.1371/journal.pntd.0001730

33. Grange L, Simon-Loriere E, Sakuntabhai A, Gresh L, Paul R, Harris E. Epidemiological risk factors associated with high global frequency of inapparent dengue virus infections. Front Immunol. 2014;5:280. http://dx.doi.org/10.3389/fimmu.2014.00280

34. Clapham HE, Cummings DAT, Johansson MA. Immune status alters the probability of apparent illness due to dengue virus infection: evidence from a pooled analysis across multiple cohort and cluster studies. PLoS Negl Trop Dis. 2017;11:e0005926. http://dx.doi.org/10.1371/journal.pntd.0005926

35. Simon-Lorière E, Duong V, Tawfik A, Ung S, Ly S, Casadémont I, et al. Increased adaptive immune responses and proper feedback regulation protect against clinical dengue. Sci Transl Med. 2017;9:eaa15088. http://dx.doi.org/10.1126/scitranslmed. aal5088

36. Li D, Lott WB, Lowry K, Jones A, Thu HM, Aaskov J. Defective interfering viral particles in acute dengue infections. PLoS One. 2011;6:e0019447. https://doi.org/10.1371/journal. pone.0019447

37. Yung C-F, Lee K-S, Thein T-L, Tan L-K, Gan VC, Wong JGX, et al. Dengue serotype-specific differences in clinical manifestation, laboratory parameters and risk of severe disease in adults, Singapore. Am J Trop Med Hyg. 2015;92:999-1005. http://dx.doi.org/10.4269/ajtmh.14-0628

Address for correspondence: Arnaud Tarantola, Institut Pasteur du Cambodge, Epidemiology and Public Health Unit, PO Box 983, Phnom Penh, Cambodia; email: arnaud.tarantola@pasteur.fr

\section{EID Podcast: Antimicrobial Drug Resistance and Gonorrhea}

Neisseria gonorrhoeae, the causative pathogen of gonorrhea, has been designated an urgent antimicrobial drug resistance threat by the Centers for Disease Control and Prevention. Since the introduction of antimicrobial drugs in the first half of the 20th century, N. gonorrhoeae has successively developed resistance to each antimicrobial agent recommended for gonorrhea treatment. In the United States, the prevalence of resistance in $N$. gonorrhoeae often varies by sex of partner and by geographic region. Prevalence is often greater in isolates from gay, bisexual, and other men who have sex with men than those from men who have sex only with women, and prevalence is often highest in the West and lowest in the South. Resistant strains, in particular penicillinase-producing $N$. gonorrhoeae, fluoroquinolone-resistant $N$. gonorrhoeae, and gonococcal strains with reduced cephalosporin susceptibility, seemed to emerge initially in the West (Hawaii and the West Coast) before spreading eastward across the country. These geographic patterns seem to support the idea that importation of resistant strains from other regions of the world, such as eastern Asia, is a primary factor of the emergence of resistant gonococci in the United States. Whereas

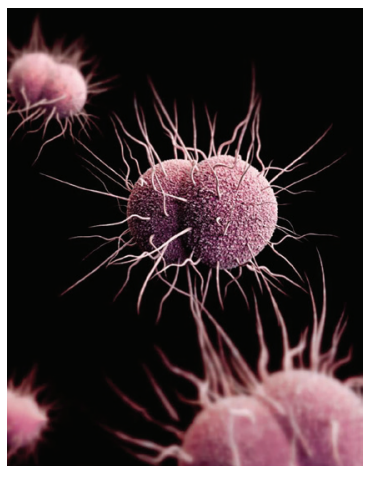
antimicrobial drug prescribing patterns have been clearly associated with the emergence of resistance in other bacterial pathogens, the degree to which domestic antimicrobial use and subsequent selection pressure contributes to the emergence of gonococcal antimicrobial resistance in the United States is unclear. Using an ecologic approach, we sought to investigate the potential geographic and temporal association between antimicrobial drug susceptibility among US N. gonorrhoeae isolates and domestic outpatient antimicrobial drug prescribing rates in the United States during 2005-2013.

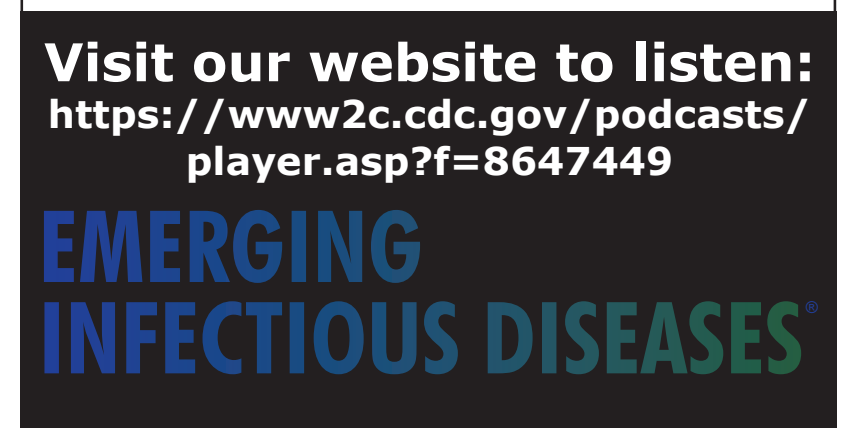

